Report LR-563

\title{
Flutter of Slender Bodies under Axial Stress
}

November 1988

R. Coene

*6\% 
DELFT UNIVERSITY OF. TECHNOLOGY

Department of Aerospace Engineering

Report LR-563

FLUTTER OF SLENDER BODIES UNDER AXIAL STRESS

R. Coene

Delft - The Netherlands

November 1988 


\section{SUMMARY}

In this report solutions of the equations of motion of flexible slender bodies with constant body sections are derived. For a certain axial stress the divergence speed and the flutter speed are obtained. The new results are compared with a classical waving flag result in two dimensional flow. The theoretical predictions compare favourably with experimental results for some paper strips. 


\section{CONTENTS}

\section{$\underline{\text { Page }}$}

\section{Summary}

List of symbols

1. Introduction

2. The equations of motion

3. Exponentially growing wave solutions

4. The two dimensional analogy

13

5. Experimental results 


\section{LIST OF SYMBOLS}

a

A

b

C

EI

$h(x, t)$

i

k

$\ell$

$\ell(x, t)$

m

$L(x, t)$

$\mathrm{p}$

$\mathrm{P}_{\mathrm{Cr}}$

$\mathrm{S}$

$\mathrm{T}$

U

V

w

$\mathrm{x}, \mathrm{y}, \mathrm{z}$

$t$

$\alpha$

$n$

$\lambda$

$\rho$

$\rho_{b}$

wave amplitude

area related to virtual mass effects of the body cross sections span of the strips

phase velocity of a wave with respect to undisturbed fluid far from the body

flexural rigidity

vertical displacements of the body sections

imaginary unit; $i^{2}=-1$

wave number

length of the body

lateral force per unit surface in the $2 \mathrm{D}$-case

mass per unit area

lateral force per unit length exerted by the fluid on the body perturbation pressure

critical load $(P=-T)$

surface area of the body cross sections

axial tension in the body

uniform flow velocity

phase velocity of a wave with respect to the body

resultant crossflow

Cartesian coordinates moving steadily with the body time

$\alpha=\rho A$ virtual mass per unit length

proportionality factor

wavelength $\left(\lambda=\frac{2 \pi}{\mathrm{k}}\right)$

density of the fluid

density of the body

velocity potential

$\sigma=\rho_{b} S$. mass of the body per unit length

circular frequency 


\section{INTRODUCTION}

At the end of chapter 15 on linearised gravity waves in [1], page 466, problems 18 and 19 involve the waves and the flapping of a two dimensional flag:

18. Two portions of a large uniform stream of liquid of density $p$, flowing with velocity $U$, are separated by a plane boundary of perfectly flexible fabric, of mass $m$ per unit area, and subject to a tension $T$, the boundary being parallel to the stream. Show that waves of length $\lambda$ can be propagated along the fabric, in the direction of the stream, with a velocity $V$ given by

$$
m V^{2}-T+\frac{\lambda \rho}{\pi}(U-V)^{2}=0
$$

provided that

$$
\mathrm{T}\left(1+\frac{\mathrm{m} \pi}{\lambda \rho}\right)>\mathrm{mU}^{2}
$$

19. Explain, giving the necessary theory, why a flag flaps in a breeze'.

This states the classical two-dimensional waving flag problem and (1.1) subject to (1.2) represents its solution.

In [4] the wave motions of a half infinite membrane placed in an incompressible fluid flow are discussed. Special attention is paid to the Kutta condition at the trailing edge and an equation is obtained for the frequency of oscillations excitated by the oncoming flow.

In the present report the slender body problem corresponding to the waving flag problem referred to above is treated. Solutions corresponding to (1.1) and (1.2) will be derived for flexible slender bodies with arbitrary cross-sections. In a private communication Gadd discussed a slender body solution for bodies of circular cross sections and neutral buoyancy under constant tension. As will be shown, these 'waving weed' solutions are readily generalized to cases with arbitrary sections without neutral buoancy. As an application of the results, the flutter speed of a strip of paper in a parallel stream will be calculated and compared with results from a series of windtunnel experiments. 
In the present context some general remarks on slender body theory are in order. Here a body is called slender if the local thickness is small with respect to the length in the direction of the undisturbed stream, in all directions perpendicular to the stream. A body whose local thickness is small in one direction only is called thin. Thus a line is the limit of a slender body as the thickness tends to zero, while the limit of a thin body is a surface. Whenever a slender body is laterally compressed the body may be both thin and slender, the result being a slender wing.

An essential feature of slender body-theory is that the velocity potential which describes the flow outside the boundary layer satisfies the two-dimensional Laplace equation in the near field. (In the far field the flow is dominated by a three-dimensional dipole). Satisfying certain conditions of smoothness in the streamwise direction solutions are readily obtained for many flow regimes of practical interest. The idea to use the concept of virtual momentum of a lateral section is due to Munk [2]. The same method is also applicable to unsteady flow problems. An interesting example of such an applicaton is due to Lighthill [3] who explains the swimming of slender fish. The theory indicates that efficient propulsion can be generated by a wave of increasing amplitude which passes down the body at a phase velocity $V$ slightly larger than the swimming speed $U$. At the sharp trailing edge a weak Joukowski condition, which allows for a finite pressure jump, is being satisfied while vorticity is being shed into the wake. Positive thrust can be generated at a positive rate of working by the body only. Conversely, a negative rate of working by the body is associated with drag. This case, of course, is related to 'flutter' and instability of motion. It will be shown that the coefficient $\frac{\lambda \rho}{\pi}$ involved in (1.1) and (1.2) can be interpreted as the 'virtual mass' for $2 \mathrm{D}$ waves.

In [7] and [8] the dynamics and the stability of towed flexible cylinders is discussed. Modes of oscillation are obtained for neutrally buoyant cylinders allowing for viscous effects and a variable tension in the bodies, keeping the nose of the body fixed in a steady stream.

Surprisingly no reference is made in [7] and [8] to the fundamental results (1.1) and (1.2) or their slender body counterparts.

The stability problem and the swimming problem are not the only ones which may lead to waves in a body. As shown in [5] a wavy stream leads to a passive recoil mode with a phase velocity equal to the phase velocity of the oncoming waves when there is no tension in the body. 
Another example is a flag waving in the von Karman vortex street of the mast, where the wave velocity is dictated by the phase velocity at which the alternating vortices are convected downstream. In this report however, we consider the free-free stability problem of slender bodies with constant tension and invariable body sections to derive the slender body counterpart of (1.1) and (1.2). Some experimental values for the flutter speeds [9] of three strips of paper will be compared with the new slender body results. 


\section{THE EQUATIONS OF MOTION}

We consider a flexible slender body in a uniform oncoming flow. A Cartesian coordinate system $(x, y, z)$ which is an inertial system has its $x$-axis aligned with the oncoming flow velocity $U$. The body has length $\ell$ and lateral dimensions which are small with respect to $\ell$ (Fig. 2.1).

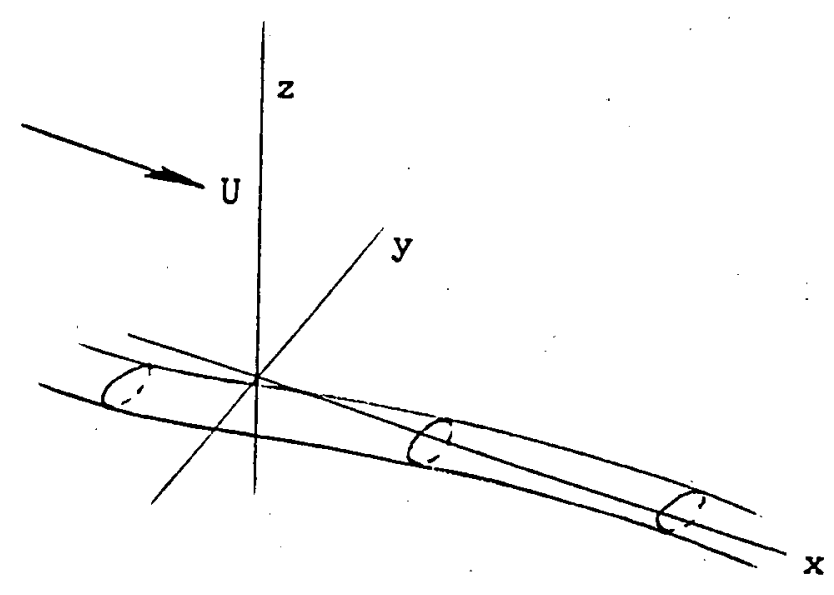

Fig. 2.1
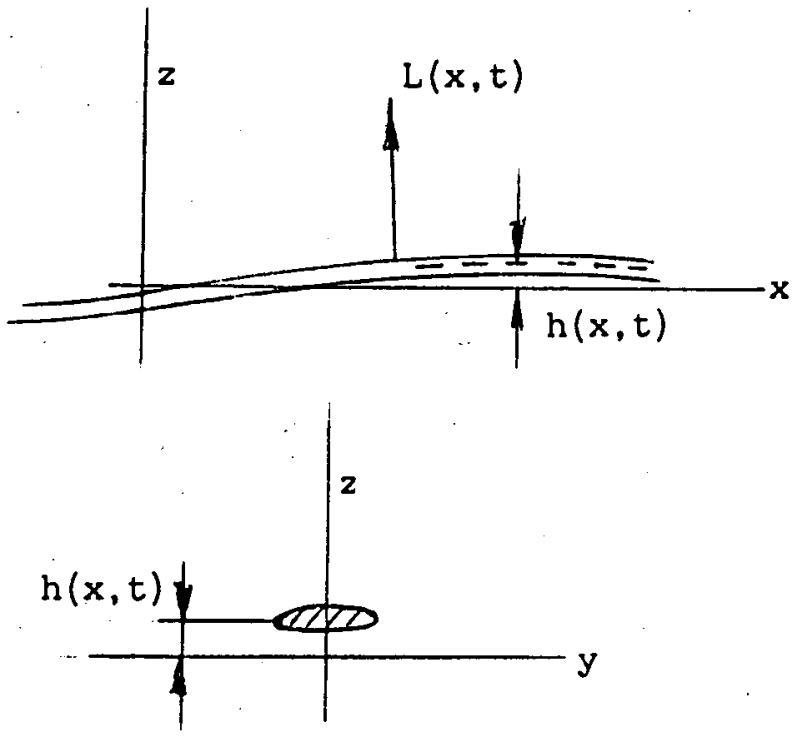

Fig. 2.2

The cross sections may perform displacements $h(x, t)$ in the vertical $z$-direction. Moreover, the $z-x$ plane is a plane of symmetry of the body (Fig. 2.2). Therefore the flow is also symmetrical and there will be no torsional moments. The z-axis is a principal axis of the body sections and we assume a bending stiffness EI. We neglect deformations due to shear.

For the lateral (z-component) force exerted by the fluid on the body per unit length in the $\mathrm{x}$-direction we use the classical slender body result

$$
L(x, t)=-\rho\left(\frac{\partial}{\partial t}+U \frac{\partial}{\partial x}\right)(w A)
$$

where $\rho$ is the density of the fluid, and $w$ is the resultant cross flow with

$$
w=\frac{\partial h}{\partial t}+U \frac{\partial h}{\partial x}
$$

$\rho A$ is the virtual mass of the cross sections (per unit length in the $x$ direction) for motions in the $z$-direction. 
The differential operator $\left(\frac{\partial}{\partial t}+U \frac{\partial}{\partial x}\right)$ appearing in $(2.1)$ and $(2.2)$ is the linearised approximation of the material derivative; i.e. a time derivation following a particle. Thus the right hand side of (2.1) stands for the material time derivative of the $z$-component of momentum of a slice of fluid of unit thickness in the $z$-direction. To the order of approximations involved one may write

$$
\frac{D}{D t}=\frac{\partial}{\partial t}+U \frac{\partial}{\partial x} \text {, }
$$

for the time derivative in a coordinate system travelling at speed $U$ in the positive $x$-direction with respect to the body. Obviously this coordinate system is fixed with respect to the fluid which is undisturbed far from the body. Equation (2.1) is valid for smooth variations in the $x$-direction and by virtue of the physical significance of (2.3) it is obvious that both $w$ and A may depend on $x$ and $t$. In this report however only $w$ is dependent on $x$ and $t$ while $A$ is assumed to be constant. This restriction is important since it implies that the expression on the right hand side of (2.1) involves only second derivatives of $h(x, t)$ with respect to $x$ and $t$.

By virtue of (2.1) the differential equation for $h(x, t)$ can be expressed as

$$
\left(\rho_{b} S\right) \frac{\partial^{2} h}{\partial t^{2}}=\frac{\partial}{\partial x}\left(T \frac{\partial h}{\partial x}\right)-\frac{\partial^{2}}{\partial x^{2}}\left(E I \frac{\partial^{2} h}{\partial x^{2}}\right)-\left(\frac{\partial}{\partial t}+U \frac{\partial}{\partial x}\right)(\rho w A)
$$

where the first three terms are the usual contributions for a vibrating beam, with

$P_{b}$ the mass density of the body,

$S$ the area of the cross sections,

$T$ the axial tension,

EI the flexural rigidity.

Assuming constant $\rho_{b} S=\sigma$, constant $E I$, constant $T$ and constant $\rho A=\alpha$, equation (2.4) simplifies to

$$
\sigma \frac{\partial^{2} h}{\partial t^{2}}=T \frac{\partial^{2} h}{\partial x^{2}}-E I \frac{\partial^{4} h}{\partial x^{4}}-\alpha\left(\frac{\partial}{\partial t}+U \frac{\partial}{\partial x}\right)^{2} h
$$


This is a fourth order linear equation for $h(x, t)$ involving only second and fourth order derivatives. This opens the way for some wave solutions of exponential growth. 


\section{EXPONENTIALLY GROWING WAVE SOLUTIONS}

Equation (2.5) has solutions of the form

$h(x, t)=a e^{i(k x-\omega t)}$.

Substitution of (3.1) into (2.5) yields a quadratic equation for $w$ (with $a \times 0$ ):

$-\sigma \omega^{2}=-T k^{2}-E I k^{4}-\alpha\left(-\omega^{2}-U^{2} k^{2}+2 \omega U k\right)$.

which is solved by

$$
\begin{aligned}
w_{1,2} & =\frac{\alpha U k \pm \sqrt{(\alpha U k)^{2}-(\alpha+\sigma)\left(\alpha U^{2}-T-E I k^{2}\right) k^{2}}}{(\alpha+\sigma)} \\
& =\frac{\alpha U k \pm \sqrt{\left(T+k^{2} E I\right) k^{2}(\alpha+\sigma)-\alpha \sigma k^{2} U^{2}}}{(\alpha+\sigma)}
\end{aligned}
$$

For real values of the wavenumber $k$ one obtains real values for $\omega_{1,2}$ with

$$
T+k^{2} E I-\frac{\alpha \sigma}{\alpha+\sigma} U^{2} \geqslant 0
$$

implying the possibility of steady oscillations.

If $(3.4)$ is not satisfied exponential growth is to be expected for

$$
T+k^{2} E I-\frac{\alpha \sigma}{\alpha+\sigma} U^{2}<0
$$

In the absence of an outside flow, the vanishing discriminant in (3.3) yields the classical Euler buckling. With $T=-P_{c r}$ and $k=\frac{2 \pi}{\lambda}$, one has its extension:

$$
P_{c r}=\frac{4 \pi^{2} E I}{\lambda^{2}}-\frac{\alpha \sigma}{\alpha+\sigma} U^{2} .
$$

For a body with neglible flexural rigidity, but also in the limit with $k=\frac{2 \pi}{\lambda} \rightarrow 0$, one obtains a unique flutter speed, $U_{c r}$ with

$$
U_{c r}^{2}=\frac{\alpha+\sigma}{\alpha \sigma} \mathrm{T}
$$


Conversely, for a given $U$ one may also define a critical tension $T_{c r}$ below which instability will arise:

$$
T_{c r}=\frac{\alpha \sigma}{\alpha+\sigma} U^{2}
$$

In the critical situation with vanishing discriminant in (3.3) the phase velocity of the waves passing down the body is given as

$$
v_{c r}=\frac{\omega}{k}=\frac{\alpha U}{\alpha+\sigma} .
$$

From (3.8) it is clear that $V_{c r}<U$.

The result (3.7) explains why the trailing part of a banner towed by an aircraft will generally be unstable. Adding a drag device behind the banner which increases the tension above the critical value (3.7a) would stabilize the banner and increase its life time. The added drag due to the extra device is not a pure loss since a flapping banner has a larger drag than a quiescent banner.

The results $(3.7)$ and $(3.8)$ can be obtained in a slightly different way which sheds some light on the physical background. Putting EI $=0$ in (2.5) and thereby retaining only the second order derivatives, equation (2.5) can be expressed as

$$
\left(\frac{\partial}{\partial t}+c_{1} \frac{\partial}{\partial x}\right) \cdot\left(\frac{\partial}{\partial t}+c_{2} \frac{\partial}{\partial x}\right) h=0 .
$$

with $\quad c_{1} c_{2}=-\frac{T-\alpha U^{2}}{\alpha+\sigma}$,

and $\quad c_{1}+c_{2}=\frac{2 \alpha U}{\alpha+\sigma}$.

From (3.10) and (3.11) one readily obtains

$$
c_{1,2}=\frac{\alpha U}{\alpha+\sigma} \pm \sqrt{\left(\frac{\alpha U}{\alpha+\sigma}\right)^{2}+\frac{T-\alpha U^{2}}{\alpha+\sigma}} .
$$

The discriminant in (3.12) vanishes with

$$
T=\frac{\alpha \sigma}{\alpha+\sigma} U^{2},
$$

and the two phase velocities are then equal:

$$
c_{1}=c_{2}=\frac{\alpha U}{\alpha+\sigma} \text {. }
$$


The flutterspeed which follows from (3.14) is the same as obtained before, in (3.7), and arises when two waves have the same phase velocity (3.15). From (3.10) we note that with

$$
\mathrm{T}=\alpha \mathrm{U}^{2}
$$

which is larger than the critical value $(3.7 a)$, one of the phase velocities, say $c_{1}$, vanishes and one finds:

$$
c_{1}=0 \text { and } c_{2}=\frac{2 \alpha U}{\alpha+\sigma} .
$$

At the special value (3.16) for the tension, a steady deformation $h(x)$, with $\frac{\partial h}{\partial t}=0$, of arbitrary shape is possible. Thus, from (3.16) we obtain a divergence speed given by

$$
\mathrm{U}_{\mathrm{div}}^{2}=\frac{\mathrm{T}}{\alpha}
$$

We note that this divergence speed is smaller than the flutter speed given by $(3.7)$. The slender body results of this section are believed to be new and in the next section they will be compared with the classical flag results (1.1) and (1.2) from [1]. 


\section{THE TWO DIMENSIONAL ANALOGY}

In the two dimensional case we start from a wave potential in the form

$$
\phi^{+}=-a c e^{-k z} \cos [k\{x-(U+c) t\}] \text {, }
$$

The + indicating the upperside of the flag. At the lower side one has $\phi^{-}=-\phi^{+}$. At $z=0^{+}$, we have for the displacements $h(x, t)$ in the $z$-direction:

$$
w=\frac{\partial h}{\partial t}+U \frac{\partial h}{\partial x}=\left(\frac{\partial \phi}{\partial z}\right)_{(z=0)}=-k \phi^{+}(z=0) \cdot
$$

For the lateral force per unit surface we have from the linearized Bernoulli equation:

$$
\ell=-2 p_{(z=0)}^{+} 2 p\left(\frac{\partial}{\partial t}+U \frac{\partial}{\partial x}\right) \phi^{+}=2 p a c^{2} k \sin [k\{x-(U+c) t\}] .
$$

From (4.2) and (4.3) we obtain

$$
\left(\frac{\partial}{\partial t}+U \frac{\partial}{\partial x}\right)^{2} h=-k\left(\frac{\partial}{\partial t}+U \frac{\partial}{\partial x}\right) \phi_{(z=0)}^{+}-\frac{k l}{2 p} .
$$

With $k=\frac{2 \pi}{\lambda}$, the lateral force may be expressed in terms of the wave length:

$$
\ell(x, t)=-\frac{\lambda \rho}{\pi}\left(\frac{\partial}{\partial t}+U \frac{\partial}{\partial x}\right)^{2} h .
$$

This leads to an equation of motion analogous to (2.5) with $E I=0$.

$$
\sigma \frac{\partial^{2} h}{\partial t^{2}}=T \frac{\partial^{2} h}{\partial x^{2}}-\frac{\Lambda \rho}{\pi}\left(\frac{\partial}{\partial t}+U \frac{\partial}{\partial x}\right)^{2} h .
$$

With

$$
\alpha=\frac{\lambda p}{\pi} \text {, }
$$

a perfect analogy with the slender body case is established. This leads to an interpretation of the coefficient $\frac{\lambda p}{\pi}$ in the two-dimensional case which is somewhat unusual. Apparently the term $\frac{\lambda \rho}{\pi}$ plays the role of a virtual mass. A layer of fluid with thickness $\frac{\lambda}{\pi}$ is effectively involved in the two dimensional flag case.

Neglecting the flexural rigidity in equation (3.2) and putting $\frac{\omega}{k}=V$, we obtain 


$$
a V^{2}-T+\alpha(U-V)^{2}=0
$$

By virtue of (4.7) equation (4.8) is equivalent to (1.1) and (1.2) is implied. We note that the slender body results of section 4 are more general than the two-dimensional result in the sense that the cross sections of the slender bodies need only be symmetrical with respect to the z-axis, while there is no need for them to be laterally compressed, or 'thin'.

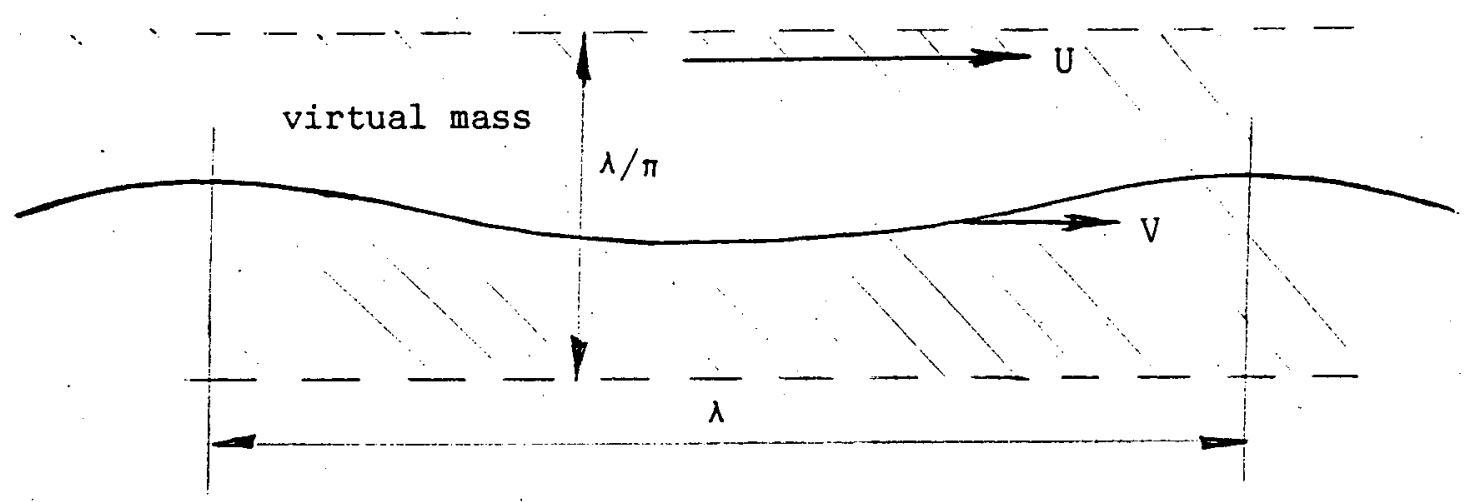

Fig. 4.1 


\section{EXPERIMENTAL RESULTS}

The results (3.7) and (3.7a) for the flutter speed and the critical tension will be compared with some experimental results for three strips of paper with different spans. These strips were mounted in the M-tunnel of the Aerospace Department in Delft, as indicated in Fig. 5.1.

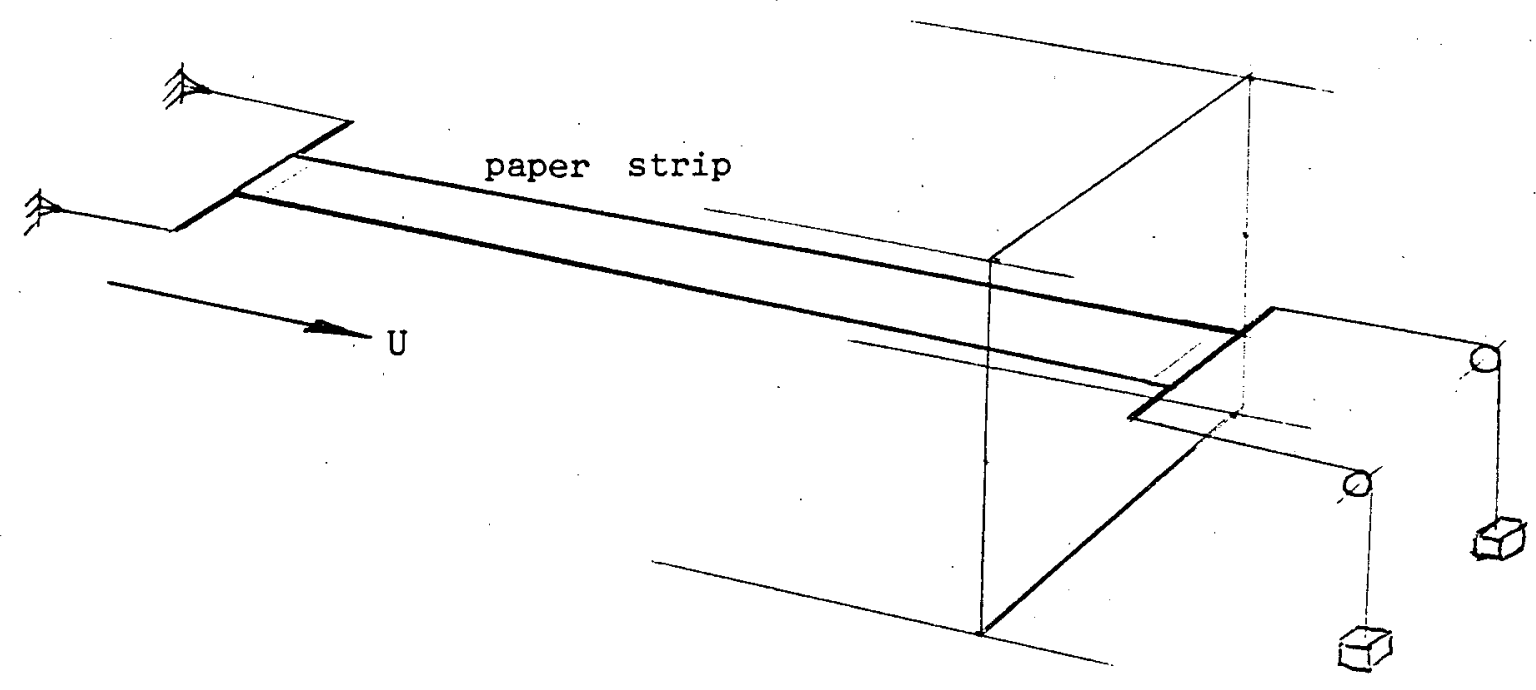

Fig. 5.1.

The end conditions for the strips were of the free-free type; the cable mountings restrained the strips only in the streamwise direction. The weights attached to the trailing edges were used to vary the tension in the strips. The tensions applied were sufficiently large with respect to the variations due to viscosity to justify the assumption of constancy of the tension. The length of the paper strips was $96 \mathrm{~cm}$. Three spans, $25 \mathrm{~cm}, 16,5 \mathrm{~cm}$ and $8,5 \mathrm{~cm}$ were used. The mass density of the paper was $1,31.10^{3} \mathrm{~kg} \mathrm{~m}^{-3}$. The flexural rigidity was considered negligible, the thickness being $0.1 \mathrm{~mm}$. The test section of the tunnel was $40 \times 40 \mathrm{~cm}^{2}$ and the speed range used was from $7 \mathrm{~m} / \mathrm{s}$ to $16 \mathrm{~m} / \mathrm{s}$, while the tension went up to $2,6 \mathrm{~N}$ for the strip of largest span.

The experiments were carried out by two students, Poland, R.Y.C.M. and Raayman, A.J.J., to whom the author expresses his gratitude. The following procedure was followed: Weights were installed to adjust the tension. Then the windtunnelspeed was increased to the point where instability was observed. Then the tension and the corresponding dynamic pressures were plotted.

The results are plotted in Figs. 5.2 and 5.3. In the latter the appropriate 


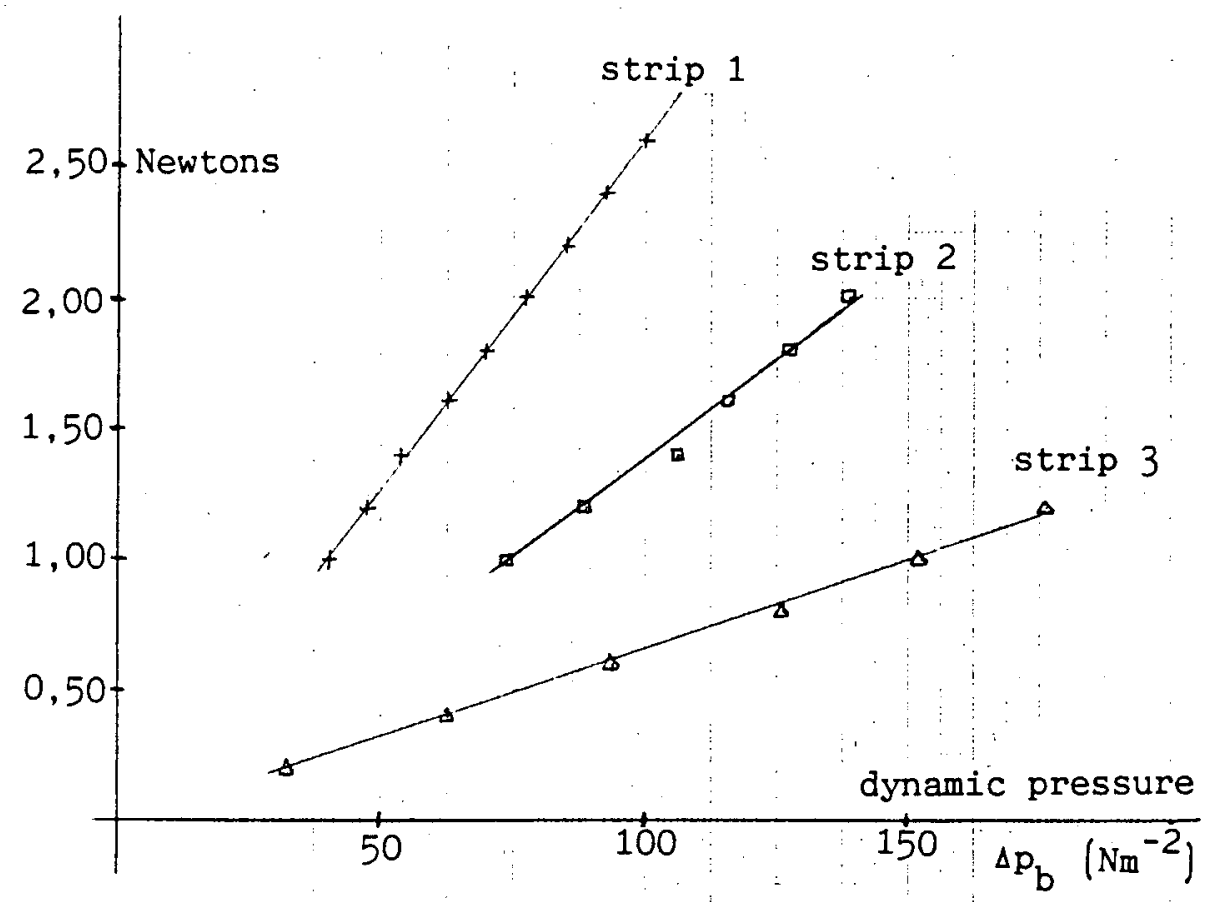

Fig. 5.2.

theoretical values are compared with the experimental values in terms of a referential tension defined by

$$
\mathrm{T}^{*}=\mathrm{U}^{2} \frac{\pi^{2} \alpha_{0} \sigma_{0}}{\pi \alpha_{0}+\sigma_{0}},
$$

where $n$ defines the span of a strip with respect to the span $b_{0}=8.5(\mathrm{~cm})$ of strip 3:

$$
b=\pi b_{0} \cdot
$$

For $\alpha_{0}$, the apparent mass per unit length of the reference strip, we have the mass of air enclosed by the circumscribed circular cylinder:

$$
\alpha_{0}=\rho \frac{\pi}{4} b_{0}^{2}=7,32 \cdot 10^{-3} \mathrm{~kg} / \mathrm{m} \text {, }
$$

and for $\sigma_{0}$ one has

$$
\sigma_{0}=1,13 \cdot 10^{-2} \mathrm{~kg} / \mathrm{m} .
$$


We note that the experimental results for strip 3 (span $b=8,5 \mathrm{~cm}$ ) are closest to the theoretical prediction. Clearly strip 3 is 'more slender' than the other two.

In a future experiment the critical phase velocity will be determined for comparison with the theoretical prediction. (3.8). It can be expected that this determination can be done in two ways: ( $i$ ) measuring the correlation in the velocity field of two streamwise positions, (ii) measuring the proportion $\left|\frac{\partial h}{\partial t}\right| /\left|\frac{\partial h}{\partial x}\right|$ by optical means.

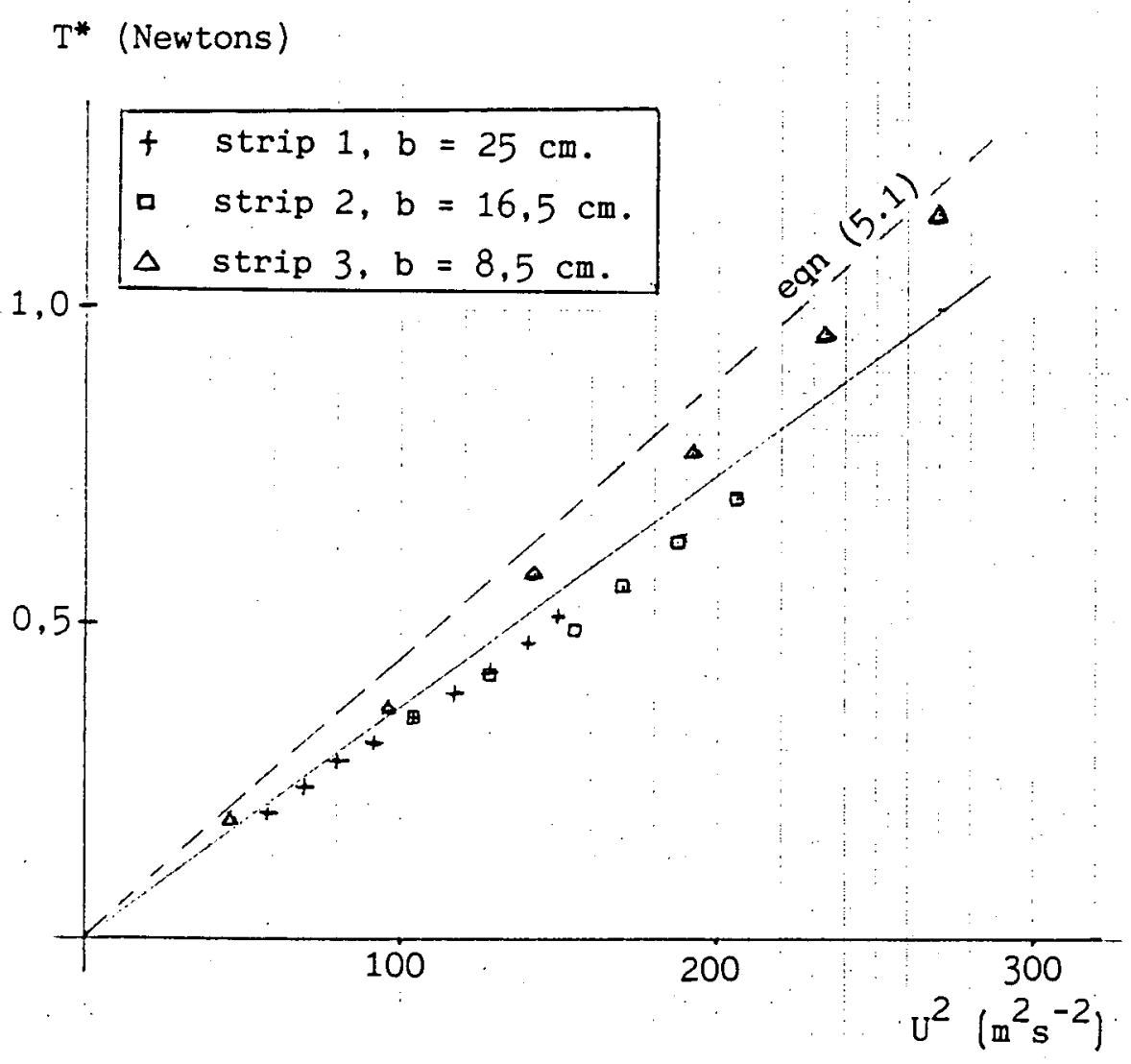

Fig. $5 \cdot 3$ : 


\section{REFERENCES}

1. Milne-Thomson, L.M.

2. Munk, M.M.

3. Lighthill, M.J.

4. Sparenberg, J.A.

5. Gadd, G.E.

6. Coene, R.

7. Paidoussis, M.P.

8. Pao, H.P.

9. Raayman, A.J.J.
Theoretical hydrodynamics.

Fifth edition (1968); London, Mac Millan \& Co.

The aerodynamic forces on airship hulls. Report 184 (1923) NACA, pp 451-468.

Note on the swimming of slender fish.

J. Fluid Mech. 9, pp 305-317 (1960).

On the waving motion of a flag.

Koninklijke Akademie van Wetenschappen (1962).

Some hydrodynamical aspects of the swimming of snakes and eels.

Philosophical Magazine, ser. 7 pp 664-670 (1952).

The swimming of flexible slender bodies in waves.

J. Fluid Mech. 72. pp 289-303 (1975).

Dynamics of flexible slender cylinders in axial flow.

J. of Fluid Mech. Vol. 26, pp 717-751 (1966).

Dynamical stability of a towed thin flexible cylinder.

J. of Hydronautics. pp 144-150 (1971).

Verslag van een experimenteel onderzoek naar een stabiliteitscriterium voor slanke lichamen geplaatst in een parallelstroming.

TZ verslag TUD, 1987. 


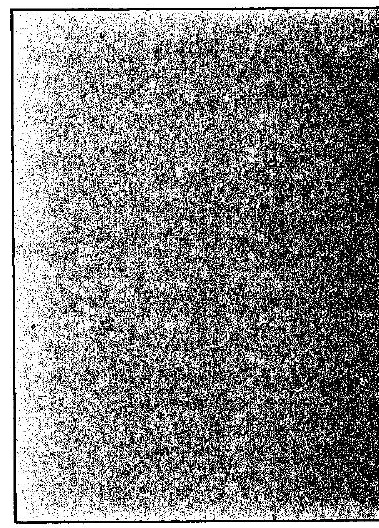

888647 